\title{
Las pequeñas y medianas empresas (PYMES) en Cumaná (Venezuela): inflación, gerencia y desarrollo
}

\author{
Ramírez de Suárez, Guillermina* \\ Solórzano Lara, Lissette $\mathbf{R}$. \\ Rosillo Canales, Yusmelis **
}

\section{Resumen}

Hoy en día, ante la grave situación socio-económica que vive el país se plantea repensar las políticas industriales cobrando especial interés el sector de las pequeñas y medianas empresas (PYMES). Tal premisa sirve de marco y motivación a la presente investigación, la cual tuvo como objetivo, el estudio de las pymes en la ciudad de Cumaná, a fin de determinar su peso en el desarrollo local, específicamente su capacidad productiva y empleadora, problemas enfrentados por la inflación y las estrategias gerenciales desarrolladas para dar respuesta a la misma, durante el período 19952000. Para el logro de los objetivos planteados, se aplicó una encuesta estructurada a los gerentes de cuarenta y ocho (48) empresas afiliadas a la Cámara de Artesanos, Pequeños y Medianos Industriales del Estado Sucre (CAPMI-SUCRE), establecidas en la ciudad de Cumaná. Los resultados señalan poca percepción de los gerentes ante la influencia de la inflación, baja capacidad empleadora, especialmente a lo que se refiere a trabajos estables, producción global por debajo de las bandas aceptadas por los organismos de seguimiento, crédito y financiamiento del sector lo que podría ser un reflejo de la recesión económica a nivel de las empresas en Cumaná.

Palabras clave: $\quad$ PYMES, inflación, empleo, producción, gerencia.

Recibido: 01-08-07 . Aceptado: 02-03-08

* Docente-investigadora del Departamento de Sociología, Núcleo de Sucre, Universidad de Oriente. Lic. En Sociología, Msc. en Planificación del Desarrollo Regional. Actualmente directora de la Escuela de Ciencias Sociales. Tele-Fax (58293) 302116.

E-mail: decanato@sucre.udo.edu.ve.

** Tesistas de la licenciatura en Sociología, colaboradoras del Proyecto de Investigación, financiado por el Consejo de Investigación de la U.D.O. 


\section{Small and Middle-Sized Companies (PYMES) in Cumana (Venezuela): Inflation, Management and Development}

\section{Abstract}

Today, faced with the socio-economic situation of the country (Venezuela), it is necessary to re-think industrial policies, and particularly in relation to small and middle-sized companies (PYMES). This was the motivational framework for this research project, the objective of which was to study PYMES in the city of Cumaná, in order to determine whether their productive and employment capacities, problems confronted with inflation, and management strategies developed during the period 1995-2000, were influential in local development. In order to measure the proposed objective, a structured survey was applied to the managers of forty-eight (48) companies affiliated with the Chamber of Artisans and Small and Middle-Sized Industries in Sucre State (CAPMI-SUCRE), in the city of Cuma ná. The results indicate the perceptions of the managers with respect to the influences of inflation, low employment Capacity, especially with reference to stable work, global production below the acceptable margins established by competent organisms, and low credit and financing levels which could reflect the economic business recession in Cumaná.

Key words: PYMES, inflation, employment, production, management.

\section{Introducción}

Para la década de los ochenta, la presencia significativa de pequeñas y medianas empresas en algunas regiones geográficas que mostraban signos de crecimiento y bienestar social, en un marco de recesión económica, obligó a repensar las políticas industriales. Estas experiencias fracturaron de alguna manera el esquema de industrialización dominante que favorecía las organizaciones de grandes dimensiones.

Esta nueva concepción se fundamentó en el dinamismo y desempeño mostrado por la pequeña y mediana empresa, que por su dimensión sufre menos que las grandes firmas, ya que su tamaño las ayuda a tener mayor capacidad de respuesta ante las presiones internas y externas (Marval, 1997:212).
Por su parte, Peñaloza (1999:374) agrega entre otros elementos que coadyuvan a la competitividad de las pymes, su flexibilidad para adaptarse a los cambios del entorno, la capacidad de respuesta para integrarse a los llamados sectores productivos sirviendo de suplidores a los restantes eslabones de la cadena, formación - aprender haciendo, la relación interpersonal entre empresarios y trabajadores y el hecho de ser cuna de verdaderos emprendedores, quienes con sus recursos limitados, pero con una gran voluntad y deseos de superación, toman en serio el reto de competir en el proceso económico.

Tales potencialidades resumen la importancia de la pyme en materia de generación de producto y de empleo, dos variables decisivas en términos de desarrollo. No obstante, las pymes desde hace casi dos décadas, han venido sien- 
do afectadas por diversos factores. Entre ellos: la forma abrupta de la aplicación de una serie de medidas de ajuste dirigidas a la apertura de la economía para elevar los niveles de competitividad y la escalada inflacionaria experimentada en el país. Tal situación, además de impactar el poder adquisitivo del venezolano, al producirse un proceso de alza continua de los bienes y servicios de la economía, les planteó el reto de crecer con inflación sin descapitalizarse en el intento.

Ahora bien, no sólo las variables macroeconómicas y en especial la inflación, tienen repercusión sobre el desarrollo de las pymes, sino también el manejo gerencial; del cual depende la eficiencia y eficacia con la que se ejecuten los lineamientos de expansión, que determinan en buena medida el avance mismo de las regiones. Ya que debemos tomar en cuenta que la gerencia, tanto pública como privada, comparten la misma importancia y responsabilidad social para el desarrollo económico del país.

Las políticas industriales debían ser asumidas por un equipo de gerentes que visualizaran la problemática que se confrontaba e implementaran las medidas necesarias para dar respuesta a la misma. En consecuencia, las distorsiones del sistema financiero y los desequilibrios macroeconómicos estimularon a los gerentes de las empresas a desarrollar conductas en el manejo de los recursos financieros, propias de la economía inflacionaria (Jaramillo, 1991:137).

Cumaná, capital del Estado Sucre, no está exenta de este proceso y evidentemente, su economía requiere de nuevas estrategias empresariales que confronten de manera acertada y competitiva la realidad económica que la circunda; entre otros retos, exige gerentes con capacidad para maniobrar sobre la base de la incertidumbre y para moverse dentro del panorama inflacionario que vive Venezuela, minimizando los riesgos que pueden llevar a sus empresas al cierre, generando con ello desempleo, informalidad y contracción del mercado, entre otras consecuencias que necesariamente afectan el desarrollo de la región. Es por ello que se trata de contribuir al desarrollo de la industria ante los niveles de informalidad y dada la importancia que tiene en materia de generación, no sólo de productos sino de empleo (Vera, 2001:66).

La situación esbozada, sirve de marco y motivación a esta investigación, de carácter descriptivo y exploratorio, planteándose como objetivo el estudio de las pymes de Cumaná, a fin de determinar su peso en el desarrollo local; específicamente su capacidad productiva y empleadora, la incidencia de la inflación sobre su desempeño y el diseño de estrategias gerenciales como respuesta a la misma, en el período comprendido entre los años 1995-2000.

A tales efectos, se aplicó una encuesta estructurada a cuarenta y ocho (48) gerentes de empresas afiliadas a la Cámara de Artesanos, Pequeños y Medianos Industriales del Estado Sucre (CAPMI-SUCRE), establecidas en la ciudad de Cumaná, y reportadas en el I Censo Empresarial realizado por dicho gremio en el año 2000. Los datos proporcionados aportaron información sustancial para el logro de los objetivos, que se espera puedan contribuir al diseño de políti- 
cas atinentes a tan importante sector de la economía.

\section{Caracterización de la PYME en Cumaná}

A los efectos de conceptualizar a las Pymes y Pymis para proceder a su clasificación, para esta investigación se ha trabajado básicamente con los conceptos emitidos por la Oficina Central de Estadística e Informática (OCEI), que toman como factor delimitador de la Pyme su capacidad empleadora (pequeñas empresas las que emplean de 5 a 20 trabajadores y mediana empresas entre 21 a 100 trabajadores). La razón que motivó tal decisión fue el hecho de que para efectos del análisis social es importante considerar de qué manera el desarrollo de las Pymes incide en el incremento del empleo y por ende en el poder adquisitivo. La seguridad social, entre otros elementos que se vinculan al desarrollo económico y social de una región o un país.

En atención a la clasificación señalada, se determinó, según la muestra estudiada, que el $83 \%$ de las empresas se pudieron considerar como pequeñas y el $17 \%$ como Medianas. Por otra parte se pudo conocer que el $50 \%$ de las Pymes se encontraban ubicadas en el renglón de las industrias tradicionales, entendidas como aquellas que se dedican básicamente a la producción de bienes de consumo destinados a satisfacer necesidades humanas de forma directa. Este grupo de empresas estaba conformado por las ramas de alimentos, bebidas, muebles, textiles, vestuarios, calzados, entre otros. El otro renglón de mayor incidencia fue el de los grupos residuales que concentraban el $24 \%$ de las empresas del sector, centrándose su producción en las ramas de tipografía e imprenta, editorial y periódicos, orfebres y joyas y las empresas de servicios. Este seguido por un $20 \%$ de las industrias mecánicas, las cuales producen bienes de capital destinados a la producción de otros bienes o de servicios y constan principalmente de maquinarias y equipos. Entre las ramas que podemos encontrar en este renglón tenemos a las industrias metalmecánicas, de aluminio, astilleros, y las industrias de construcción naval. De igual forma se encontró que apenas un $6 \%$ del total de las instalaciones, estuvo representada por las industrias intermedias, dedicadas a la producción de bienes intermedios, es decir, productos que deben ser sometidos a nuevos procesos de transformación para alcanzar su destino final. Entre ellas se encontraron las industrias químicas, de insumos para la construcción, productos plásticos, industrias básicas de hierro y acero, entre otros.

Es interesante resaltar, como dentro de la producción, el $32 \%$ de las empresas se ubicaron en la rama productiva de alimentos, la cual se constituye en la actividad de mayor representatividad, seguida por el sector de los servicios que engloba el $18 \%$ del total de la actividad productiva y por un 12\% de la producción metalmecánica.

\section{Capacidad empleadora de la pyme}

Del $17 \%$ que comprende el total de las medianas empresas en Cumaná, el $64 \%$ de las mismas empleaba durante los años 1995-1996 más de 51 trabajadores (ver Gráfico 1), disminuyendo considerablemente en 1997 y años subsiguientes, donde el porcentaje no superó 
el $37 \%$. Similar comportamiento registraron las empresas que empleaban entre 21 y 30 trabajadores, que pasa de $37 \%$ en 1995 a $26 \%$ en 1996 subiendo nuevamente en 1997 y 1998 a $37 \%$ y cayendo a $18 \%$ entre 1999 y el primer bimestre del 2000. A diferencia de las empresas que emplearon de 31 a 50 , que experimentaron un continuo aumento, pasando de $17 \%$ en 1996 a $39 \%$ para el primer bimestre del 2000.

Entre 1999 y el primer bimestre del 2000 esta situación tiende a mejorar, ya que aumenta a $38 \%$ las medianas empresas que contrataban entre 31 y 50 trabajadores. El 12\% restante no suministró información sobre el número de trabajadores con el cual contaba. Estos porcentajes marcarían la diferencia que nos haría evaluar cuan realmente positivo fue el sensible incremento que tuvo la oferta de empleo en el ámbito de las medianas empresas, en la ciudad de Cumaná.

En el caso de la pequeña empresa (Ver Gráfico 2), el proceso de empleo se fue inclinando hacia la disminución del personal ocupado. Si bien aumentó el número de empresas que empleaban entre 1 a 10 trabajadores, no se observó incremento de empresas que emplearan entre 11 a 20. Por el contrario disminuyeron de $30 \%$ para 1995 a $26 \%$ para el primer bimestre del año 2000.

Ahora bien es interesante observar que hay porcentajes significativos de gerentes que no suministraron información, esto pudo estar motivado bien a que la compañía haya estado inactiva en ese período, o que existe una real negativa al respecto, ya que como sostiene Mary Vera (2001:80), la disponibilidad de la información contable y financiera a nivel interno está restringida a la alta dirección, pues este tipo de registro se considera confidencial.

Las cifras anteriores indican básicamente que la capacidad empleadora real de la pyme en general en la ciudad de Cumaná, ha ido disminuyendo paulatinamente desde 1995 hasta el año 2000, ya que aún cuando se puede hablar de au-

\section{Gráfico 1}

Porcentaje de personal empleado en la mediana industria en Cumaná (Venezuela) año 1995 - primer bimestre 2000

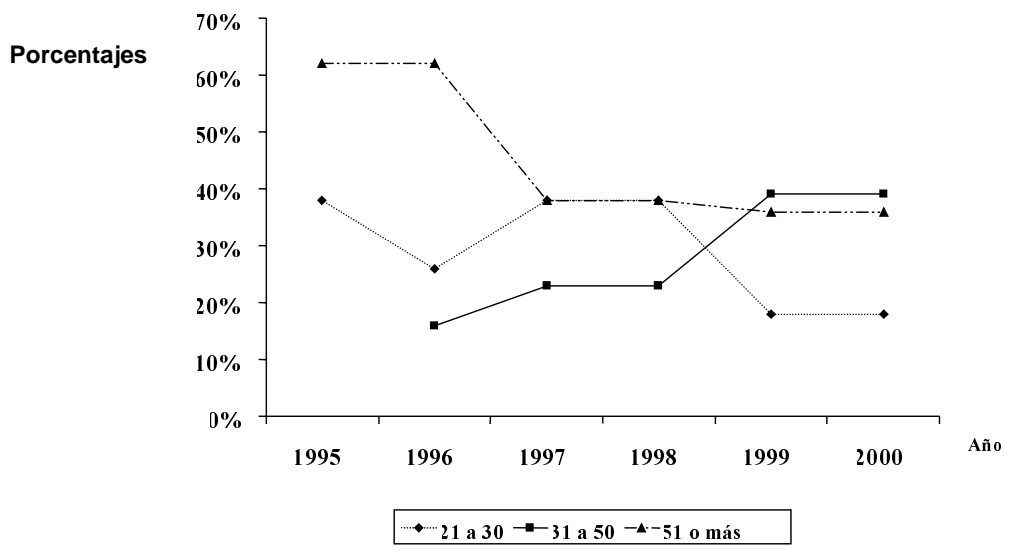

Fuente: Solórzano Lara y Rosillo, 2000 


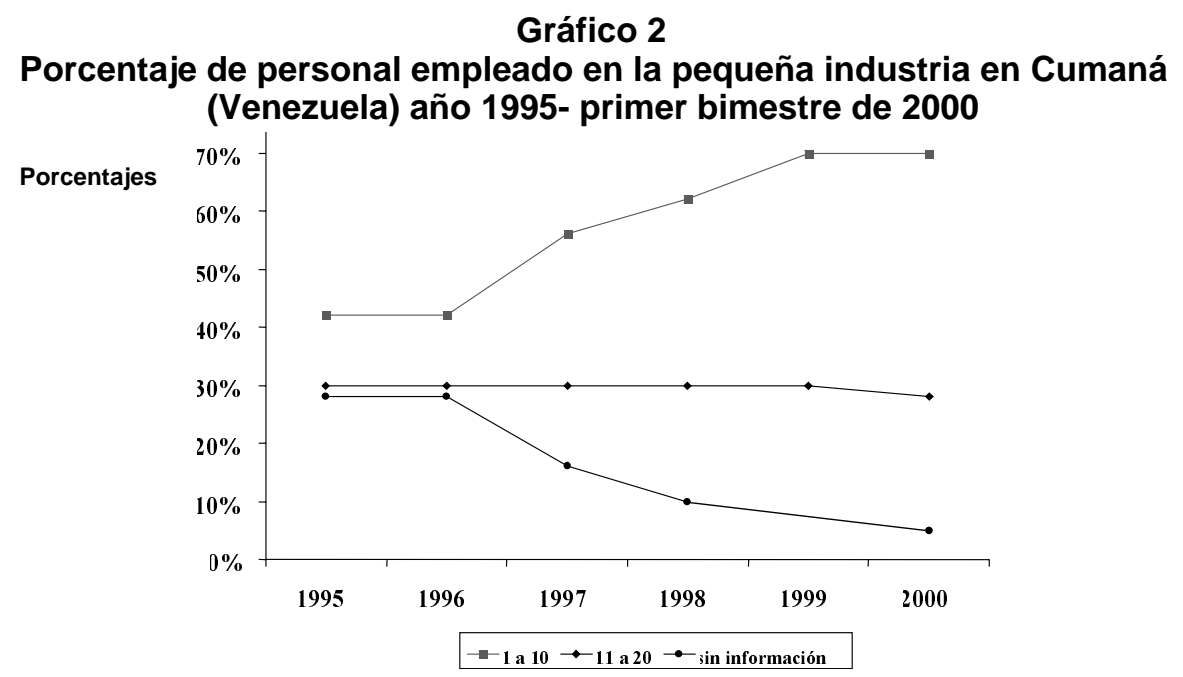

Fuente: Solórzano Lara y Rosillo (2000).

mento en la cantidad de puestos de trabajo ofertados, debido a la apertura de nuevas pymes, no se puede decir que ha habido un aumento real del trabajo, ya que por un lado se emplearon menos trabajadores o se contrató personal a destajo con la intención de abaratar costos. En otras palabras la mayoría de las pymes han reducido la cantidad de trabajadores como forma de respuesta a la situación económica que confrontan; especialmente a partir del año 1997, inmediato al año donde la inflación experimentó valores de tres dígitos, según información del Banco Central de Venezuela y justamente un año después de la implementación de las políticas de ajuste contempladas en la Agenda Venezuela, impulsada por el presidente Rafael Caldera.

Es evidente que la disminución de puestos de trabajo fijos en las pymes, es decir, trabajo formal con seguridad social, prestaciones e ingresos estables, incidirían en un aumento de la probabilidad de que más personas se sumen a las filas de los trabajadores informales, lo que podría llevar a un incremento en los índices de pobreza.

De acuerdo a información suministrada por Hernández (2000: E-1), si el empresario decide asegurar el pago de 10 trabajadores por la vía del financiamiento bancario debería solicitar $200 \mathrm{mi}$ llones de bolívares para cancelarlos en 12 meses a una tasa anual de $38 \%$. Únicamente por concepto de intereses, el desembolso sería de 76 millones de bolívares adicionales. Es por ello que la inflación comienza a incidir negativamente en las finanzas de la empresa, pues lograr la meta inflacionaria prevista afecta a los deudores bancarios ya que el sistema bancario en aras de evitar un exceso de flujo monetario presiona sobre los intereses de los créditos. Es decir, los procesos inflacionarios tanto de alza, como los de baja, en parte son producto de políticas económicas reguladoras del Estado que presionan hacia arriba los intereses de los créditos bancarios; esto afecta direc- 
tamente a las empresas, más aún a las pymes cuya capacidad de respuesta ante los altos niveles de interés bancario es menor, imposibilitándoles el acceso al financiamiento bancario de carácter privado, y en muchos casos a la renegociación de deudas pasadas (Hernández y otros, 2001:135). A ello se pueden sumar otras acciones encaminadas a disminuir el índice inflacionario, lo cual podría ser el producto de una contracción en la demanda y por ende una disminución de los gastos, haciéndose cada vez más compleja la situación financiera de los pequeños y medianos empresarios. En el mismo sentido, Fernández y Borgucci (2001:97) señalan la necesidad de examinar más los proyectos de inversión para los cuales se solicita el crédito, de manera que la banca no fundamente su decisión de otorgar prestamos en función únicamente de los balances financieros.

La contracción en la demanda genera a su vez contracción en la producción y al mismo tiempo en las ventas, lo cual trae como reacción una disminución en la solicitud de créditos y es que "las reducciones de los niveles de endeudamiento, donde la disponibilidad de recursos lo permitía, era una acción esperada, ya que muchas industrias (...) habían hecho un uso extensivo de endeudamiento mientras las tasas de interés eran negativas. Una vez que se empezó a corregir este desequilibrio, no era rentable mantener tal nivel de endeudamiento" (Jaramillo, 1991: 141). Ello obliga a los empresarios a modificar los canales de distribución, realizando una comercialización más directa y continua, acortando los días-calle de las cuentas por cobrar; también a reducir los gastos de nómina de la compañía.

\section{Potencialidad productiva de la pyme}

Para el año 1998, y según lo establecido por FEDEINDUSTRIA, una pequeña empresa debería facturar anualmente entre 100 y 200 millones de bolívares, por su parte SOGANPI considera que una pyme debe tener ventas anuales mínimas de 14 millones y máximas de 1.880 millones de bolívares. Tomamos como referencia estas dos concepciones sobre las bandas en las cuales debe moverse el mínimo y el máximo de la producción anual en bolívares de la pyme, por concepto de ventas; observando el Gráfico 3 , podemos constatar que a nivel productivo, la mayoría de las medianas empresas se habían movido, en los cinco años en estudio, dentro de los márgenes establecidos por SOGANPI. Con excepción de 1995 , no menos del $60 \%$ de las medianas empresas habían tenido una producción anual que oscilaba entre 100 y 250 millones de bolívares y con excepción del año 1996 no menos del 12\% había tenido una producción de 650 o más millones de bolívares al año. Para 1999 la producción percibió un leve crecimiento ya que el $36 \%$ de las medianas industrias registraron una producción que osciló entre 250 y 400 millones de bolívares.

En el ámbito de la pequeña empresa, como se observa en el Gráfico 4, de acuerdo a los índices productivos señalados, no se alcanza el mínimo de la producción anual que debe tener la misma, pues sus ventas no ascienden a más de 10 millones de bolívares anuales, con excepción de los años 1998 y 1999, cuando un $8 \%$ de los gerentes manifestaron que su producción estaba por el orden de los 30 y 40 millones de bolívares anuales. 


\section{Gráfico 3}

Porcentaje de empresas y Producción en Bolívares de las ventas de la pequeña industria en Cumaná (Venezuela) año 1995- primer bimestre 2000

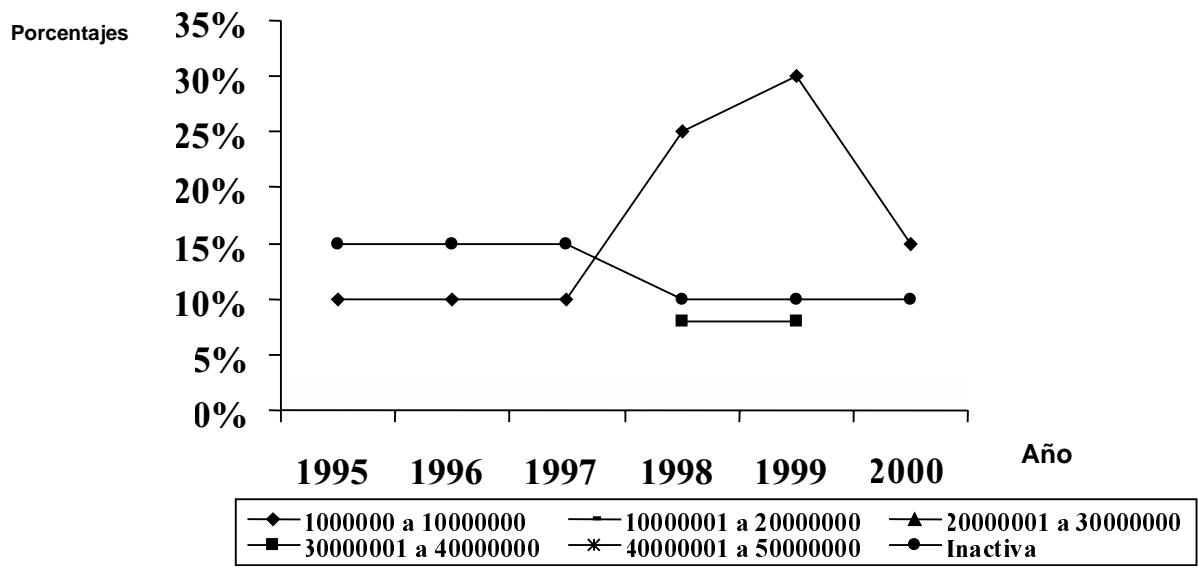

Fuente: Solórzano Lara y Rosillo (2000).

\section{Gráfico 4}

Producción en Bolívares de las ventas de la mediana industria en Cumaná (Venezuela) año 1995- primer bimestre 2000

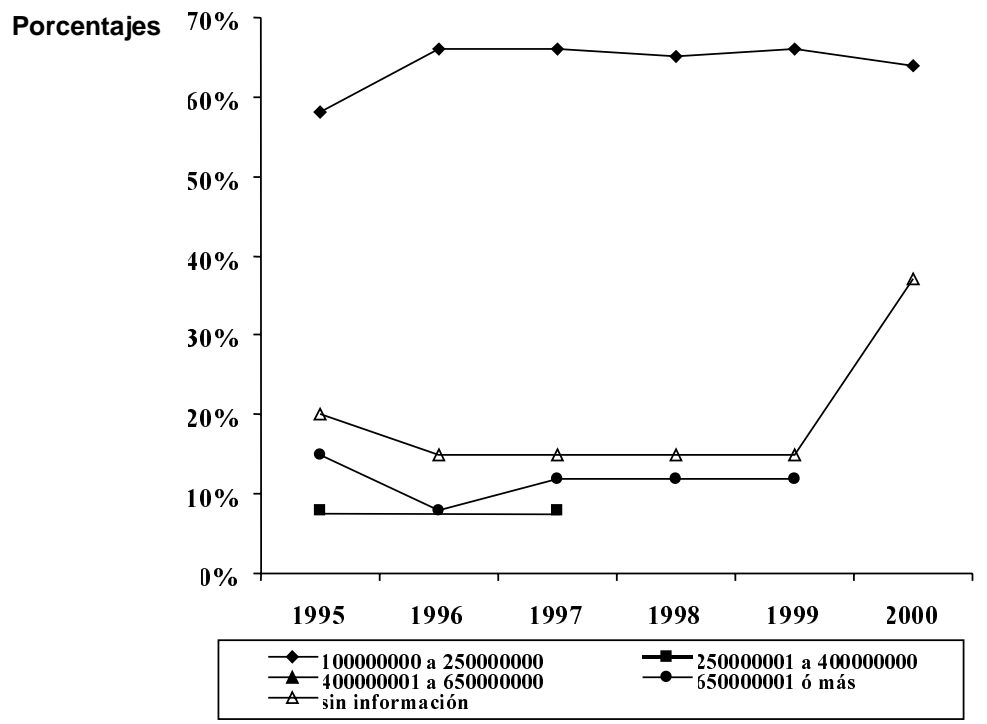

Año

Fuente: Solórzano Lara y Rosillo (2000). 
Del análisis anterior pudiera derivar que la producción global de la pequeña empresa está por debajo de las bandas establecidas lo que podría ser el reflejo de una recesión económica a nivel de la producción empresarial en Cumaná.

\section{Las pymes ante el proceso inflacionario}

En teoría la relación entre la pyme y la inflación se evidencia fundamentalmente cuando se mide el grado de desarrollo empresarial de un país y se relaciona con los niveles de inflación vividos por el mismo en períodos determinados. En este sentido, no podría entenderse dicho proceso, si no se vincula con las variables macroeconómicas endógenas y exógenas que intervienen en el mismo; tales como: el déficit fiscal, los índices de producción, los niveles de exportación e importación y la inflación entre otros indicadores de la actividad económica de una región.

En atención a esta relación señala Maza Zabala (1992:254), que la inflación, devaluación y déficit fiscal constituyen una tríada que gravita profundamente sobre la sociedad venezolana con mayor impacto en una economía importadora como la nuestra, con tradición de protección del sector industrial. Donde el mayor costo en bolívares de los bienes y servicios importados inciden en el aumento de los costos de producción y operación en el país y en consecuencia en el alza de los precios. Todo ello sin propiciar condiciones favorables para la producción sustitutiva de importaciones y poco para el impulso a exportaciones no tradicionales. De allí que la inflación hace perder competitividad a la economía, al afectar las tasas de interés bancario, la devaluación de la tasa de cambio, el alza en las tarifas de los servicios productivos, los incrementos en los sueldos y salarios, las alzas en los precios de la materia prima entre otros.

De manera que no sólo la población se ve afectada por la inexistencia de lo que podría denominarse una cultura inflacionaria, lo cual se explica por el hecho de que los venezolanos hasta hace unos veinticinco años no tuvimos necesidad de generar mecanismos de defensa contra la inflación, el sector industrial era por igual víctima de ello y a su vez de la ausencia de una política productiva eficiente (Maza Zabala, 1996:277). De allí que al vernos enfrentados a un panorama inflacionario y recesivo, con cambios en los hábitos de consumo, ahorro e inversión, el sector empresarial sufre un impacto de gran magnitud.

En atención a lo anterior, Cardoso (1991:47) agrega: la inflación amenaza el crecimiento sostenido; la incertidumbre inflacionaria acorta los horizontes para las decisiones de producción y genera preferencia en aquellos activos que protegen contra la inflación. La estructura económica, enfatiza las finanzas a expensas de la producción. La consecuencia es la fuga de capitales.

Estas afirmaciones se reflejan en la economía sucrense, pudiéndose conocer de acuerdo a la información suministrada por los gerentes de las pymes, que la contracción de las ventas fue el principal problema generado por la inflación (45\%). Se observa en líneas generales, que a los gerentes les resultaba difícil diferenciar los problemas ocasionados por el fenómeno inflacionario, de aquellos causados por cambios en la actividad económica del país. Debemos recordar que no todo proceso inflacionario tiene que producir 
una contracción de las ventas; ésta contracción no sólo depende de cambios en los patrones de consumo por la caída del ingreso real de la población, también depende de cambios en la demanda de bienes y servicios por parte de los sectores económicos, como respuesta al volumen de actividades esperadas en los próximos meses.

No obstante, la iliquidez (30\%) y la necesidad de capital $(30 \%)$ fueron los problemas que recibieron mayor atención de los gerentes, pues ello evidentemente lleva a una descapitalización de la compañía (25\%). Los problemas relacionados con el aumento de cuentas por cobrar (15\%), problemas salariales $(14 \%)$ e incluso el aumento en el pago de servicio e impuestos (15\%) recibieron una menor atención. Esto pudiera explicarse, porque debido a la crisis originada por la inflación y otros factores macroeconómi $\cos$, las funciones de tesorería cobraron mayor importancia. Tales como: política de crédito y cobranzas, estimación de ingresos y desembolsos y de los flujos de efectivo, financiamiento y colocación a corto plazo, ma nejo de operaciones bancarias, políticas para asumir niveles de riesgo, centralización/descentralización de la toma de decisiones y el nivel de automatización de los procedimien tos señalados (Vera, 2001:73).

Debido a ello, los gerentes optaron por prácticas agresivas en el manejo del dinero. Un grupo sugirió cambio en las condiciones de ventas, básicamente en lo referido a los montos y los plazos de pago, más sin embargo, la mayoría de ellos tuvieron que aumentar el tiempo en los plazos de cobro debido a la recesión. La iliquidez en este caso favoreció el aumento en las cuentas por cobrar, pese a que la tendencia expresada por los gerentes apuntaba a reducir los días-calle de las mismas para evitar que incrementara su morosidad.

De allí que el mayor porcentaje (68\%) de los entrevistados consideró que la producción fue el área más afectada por el proceso de inflación, seguido de las ventas (64\%), y es que al disminuir las ventas se desestimula la producción, y no necesariamente, por una disminución de capital. Ahora bien en el caso de la inversión, encontramos que la disminución de la misma es una conducta propia asumida por cualquier compañía en economías inflacionarias; de hecho se observó que la inversión fue la cuarta área con mayor porcentaje de afectación (44\%), lo cual ratifica el hecho de que la inversión disminuye significativamente en economías inflacionarias, como conducta natural de las compañías venezolanas, que se pliegan por "las actividades a corto plazo y las de índole financiero (...); asimismo se reducen las expectativas y los niveles de inversión" (Jaramillo, 1991:147).

Al evaluar el nivel de afectación que ha tenido la inflación en la pyme, se encontró que el mismo, por centrarse en áreas tan importantes como son las de producción y financiamiento (48\%) básicamente, es evidentemente alto. Sobretodo en el ámbito de las pequeñas empresas, y no sólo por el hecho de poseer el mayor porcentaje de instalaciones industriales, sino por tener una baja disponibilidad de recursos propios y poco acceso al crédito, por lo cual la inflación los golpea más fuertemente a nivel financiero, más aún si tomamos en consideración el restringido acceso al crédito bancario del cual disponen.

Otro aspecto interesante de destacar es que la mayoría de los entrevistados le dieron mayor importancia a los pro- 
blemas de corto plazo, como la contracción de las ventas, o la iliquidez, que a problemas de largo plazo como la descapitalización. Ahora bien, esto puede deberse a que los gerentes de las pymes tengan poco conocimiento del efecto erosivo de la inflación sobre la estructura patrimonial de la compañía, por no aplicar la variable de ajuste por inflación en sus cuentas, o que la delicada situación por la que atraviesan los gerentes y sus compañías los obligue a focalizar sus esfuerzos a resolver problemas a corto plazo.

\section{Las pymes y la gerencia: el verdadero desafío}

El proceso de globalización de los mercados, aunado a la evolución de las tecnologías de información ha generado una serie de transformaciones en la forma de hacer negocios y en la manera de conducir una empresa. Todo ello ha determinado un cambio en lo que es la gerencia actual. De aquí la importancia del establecimiento de estrategias gerenciales innovadoras, que permitan afrontar los procesos de cambio y competir en un entorno plagado de indefiniciones e incertidumbres.

A lo interno del país, variadas circunstancias obligan en muchos casos al cierre de empresas, poco uso de la capacidad instalada y baja participación en las exportaciones. Tal situación amerita la sinceración del aspecto financiero y del tejido organizacional de la pyme. Los gerentes por su parte, como opciones prioritarias, tienden a hacer uso de técnicas financieras para lograr la reducción de costos y el mantenimiento de la utilidad bruta. Ahora bien para los gerentes de las empresas en estudio, reducción de costos y políticas de ahorro se convirtieron en símiles de reducción de nómina y disminución de la producción. En los resultados arrojados por las encuestas se pudo observar que 27 de las 48 industrias, es decir el equivalente al $56,25 \%$, consideraron que la estrategia más inmediata ante la inflación era precisamente reducir el número de trabajadores y la producción de la empresa. Lo cual puede entenderse, si relacionamos tal conducta con los principales problemas detectados por ellos como generados por la inflación y la forma como afectó a la industria.

En otro sentido, se observó que la transferencia y diversificación de actividades, la inversión cooperativa, la apertura a nuevos mercados e incluso la reinversión, fueron estrategias que recibieron menor importancia. Menos del $40 \%$ de los gerentes consideraron que esas serían mejores estrategias como respuesta a las influencias del entorno. Ahora bien, esta no es una situación exclusiva de Cumaná, por el contrario es una actitud que se corresponde con el común en casos similares, ya que ante un panorama recesivo e inflacionario los gerentes recurren a estrategias de menor riesgo, para garantizar sus inversiones.

Tal actitud no necesariamente debería incluir el factor inversión, más aún cuando la búsqueda de nuevas áreas de negocios es una típica respuesta en período de crisis económica, en los cuales se esperan cambios importantes en los patrones de consumo de las personas (Jaramillo, 1991:144). Por tanto, llama la atención que para nuestros empresarios ésta no sea una de las respuestas más idónea, por lo cual se puede inferir que el mercado potencial es muy limitado o que existe en los gerentes de la pyme una predisposición al riesgo de invertir. 
La gerencia de la pyme en Cumaná necesita de acciones más contundentes a nivel de inversión, comercialización y diversificación de la producción, o en otras palabras menor temor al cambio, ya que para muchas de ellas no asumir estrategias adecuadas casi significaría la respuesta precisa para evitar el cierre y la descapitalización total. De hecho, los gerentes que asumieron estrategias como diversificación de la producción, lograron resolver problemas puntuales como el flujo de caja y pago de nómina. Muchos de ellos manifestaron que si bien no habían abierto nuevos empleos, tampoco habían prescindido de ninguno de sus trabajadores; por tanto es lógico pensar que el resto de los empresarios asumieron la vía de escape rá pida: menor producción, menos empleos e igual ganancias.

Por tanto, quizás las críticas que se le hacen a la empresa, no sean del todo irrazonables, ya que ciertamente los mecanismos utilizados privilegiaron la mantención del nivel de ganancia, que para muchas durante años, han sido nefastas. Y aún cuando ciertamente el panorama económico limita la inversión debido al nivel de riesgo, también es cierto que la salida a la crisis no se consigue sin sacrificios necesarios de parte y parte.

Ahora bien, esta actitud de los empresarios frente a la inversión, no se corresponde con el perfil ideal del buen gerente que ellos diseñaron en la encuesta, el cual tiene entre sus primeras tres cualidades el ser innovador (80\%) competitivo (78\%) y decidido $(72 \%)$ además de creativo, flexi ble, visionario, estricto, dinámico, disciplinado, responsable y cooperativo.

En su mayoría, las pymes tienen como debilidad la poca incorporación de tecnologías, modernización y asistencia tecnológica, así como limitaciones organizativas, financieras e insuficiencias gerenciales que impiden hacer uso óptimo de las capacidades humanas y equipamiento disponible (Fedeindustria, 1989: 13). Se puede notar que muchas de ellas están vinculadas a la gerencia y manejo de información; no obstante tales limitaciones, el verdadero desafío de los gerentes será convertir las mismas en fortalezas, en la medida que enfrenten exitosamente el clima inflacionario del país y así impulsar el desarrollo empresarial del mismo.

\section{Conclusiones}

Considerando los aspectos tratados, se puede inferir, en términos globales que la pyme en Cumaná y en particular la pequeña empresa pudiera estar ante una situación difícil, no sólo debido a la caída de la producción y la disminución de las ventas, sino a la falta de visión gerencial que le permita ser más competitiva. No existe una clara percepción de la influencia de la inflación y de otros factores macroeconómicos en el mediano y largo plazo; pocos gerentes propusieron cambios sustanciales orientados al aumento y diversificación de la producción; como respuesta coyuntural a los problemas mencionados optaron por reducir los niveles de inversión.

Dado que el número de grandes empresas ubicadas en Cumaná y en el Estado Sucre en general no es significativo, las consecuencias derivadas de las consideraciones anteriores, podrían afectar a un grueso de la población, ya que la baja en la producción conlleva el despido de trabajadores y en consecuencia a un aumento del desempleo, que es uno de los factores que estimula el crecimiento del sector informal 
de la economía y aumenta los niveles de pobreza. Tal situación requiere ser atendida con urgencia por los organismos gubernamentales, a través de políticas y programas de apoyo; pues a pesar de las debilidades de la pyme en Cumaná, como sector constituye un gran potencial para el desarrollo regional.

Es por ello que, aún cuando debemos admitir que el crecimiento económico no garantiza el desarrollo social por factores como el uso de mano de obra, la tecnificación de los procesos productivos, los altos índices de ganancias de las industrias, entre otros, no es menos cierto que el mismo es un elemento importante en cualquier proceso de desarrollo que se lleve a cabo en el país o en la localidad.

El carácter exploratorio del estudio, no permite llegar a conclusiones definitivas, más bien sugiere futuras investigaciones, enfocadas a renglones y ramas particulares, ya que el desempeño del sector depende de la actividad industrial específica.

\section{Bibliografía Citada}

Cardoso, Eliana (1991), "Inflación: el caso de Brasil y México". En Frances, Antonio y Dávalos, Lorenzo (compiladores) Inflación, Empresa y Sociedad. Caracas. Ediciones IESA. (47-71).

Fedeindustria (1989), "La Reconversión industrial y el proceso de exportaciones no tradicionales: Posición de la artesanía, pequeña y mediana industria". XVIII Congreso. Cumaná, Venezuela.

Fernández, Peggui B. y Borgucci, Emmanuel (2001), "El crédito bancario y la pequeña y mediana industria metalmecánica del municipio Maracaibo" En Revista de Ciencias Sociales Vol. VII, No. 1, Enero-abril. Maracaibo. LUZ. (90-111).
Hernández, Lissette y otros (2001), "La fuente de financiamiento a corto plazo y su administración en la pequeña y mediana industria (PYMI) en la región zuliana. (Sector confección, 19981999)" En Revista de Ciencias Sociales Vol. VII, No. 1, Enero-abril. Maracaibo. LUZ. (112-137).

Hernández, Vladimir (2000), "Pymis pagan intereses de Bs.3,8 millones por cada Bs. 10 millones que solicitan créditos". Diario EI Nacional, Cuerpo E-1. Venezuela.

Jaramillo, Carlos (1991), "Cambios de las prácticas financieras en períodos Inflacionarios". En frances, Antonio y Dávalos, Lorenzo (compiladores). Inflación, Economía, Empresa y Sociedad. Cáp. 7. Caracas. Ediciones IESA,. (137-163).

Marval, Oscar (1997). "Importancia y desarrollo de la pequeña y mediana industria dentro de la economía venezolana período 1981-1991". En Aranda, Sergio, Fajardo Víctor y Luis Mata Compiladores). El desarrollo económico como problema.Serie Foro al Día No 2. Caracas. Cendes, (209-220).

Maza Zabala, Domingo (1996). Crisis y política económica. 1989-1996. Caracas. Editorial Metrópolis, Cáp. 8 (261-290).

Peñaloza, Marlene (1999) "Importancia y desempeño de la pequeña y mediana industria (pymi) dentro de la economía venezolana 1989-1996". En Damacena, Claudio y José Barreiro (Compiladores). VIII International Conference European Association of Management and Business Economics. Brasil. Editorial Unisinos y AEDEM.

Vera Colina, Mary (2001) "Gestión Financiera de la pequeña y mediana industria en la ciudad de Maracaibo". En Revista de Ciencias Sociales Vol. VII, No. 1, Enero-abril. Maracaibo. LUZ. (65-89). 